\title{
Efficient AMP decision and search range adjustment algorithm for HEVC
}

\author{
Xinpeng Huang ${ }^{1,2}$, Ping $A n^{1,2^{*}}$ and Qiuwen Zhang ${ }^{3}$
}

\begin{abstract}
The advanced video encoder High Efficiency Video Coding (HEVC) utilizes several novel coding tools so that it can obtain improvement in coding performance for a huge number of video data. However, these tools increase the computational complexity greatly specially in the interprediction phase. Therefore, optimization for interprediction plays an important role in accelerating the whole HEVC encoding process. To this end, this paper proposes an efficient prediction algorithm for improving HEVC intercoding. According to the spatial correlation between the current block and its co-located block in the previous frame, we design a fast decision method so as to perform asymmetric partitions directly without the complicated calculation. Furthermore, based on the motion degree function we defined, the motion search range of motion estimation can be adaptively adjusted. Experimental results demonstrate that the proposed algorithm is able to save coding time significantly both under RandomAccess and Lowdelay P configurations, with a negligible drawback in term of Bjontegaard delta-rate increase.
\end{abstract}

Keywords: HEVC, Interprediction, Asymmetric partition, Search range

\section{Introduction}

High Efficiency Video Coding (HEVC), an advanced video coding standard, is a good substitute for encoding ultra-high-definition video. In contrast to other standards, HEVC employs several new coding tools to eliminate redundancy existing in inter- and intraframes [1-4]. The higher precision and more flexible partition structure is one of the major reasons that HEVC outperforms H.264/AVC [5]. Additionally, good performance of HEVC makes it be applied to several extensions [6-11]. In HEVC, every frame of video is divided into coding tree units (CTUs) and each CTU can be split recursively using quad-tree structure, until the minimum size is reached. Similar to macroblock concept in H.264/AVC, coding unit (CU) is the basic unit in HEVC that carries the whole coding information of current block $[12,13]$. There are eight prediction units (PUs) shown in Fig. 1, and these eight PU partitions are named as PART_2N $\times 2 \mathrm{~N}$, PART_N $\times \mathrm{N}, \mathrm{PART} 2 \mathrm{~N} \times \mathrm{N}$, PART_N $\times 2 \mathrm{~N}, \mathrm{PART} 2 \mathrm{~N} \times \mathrm{nU}$, PART_2N $\times \mathrm{nD}$, PART_nL $\times 2 \mathrm{~N}$,

\footnotetext{
* Correspondence: anping@t.shu.edu.cn

${ }^{1}$ Shanghai Institute for Advanced Communication and Data Science,

Shanghai University, Shanghai 200444, China

${ }^{2}$ Key Laboratory of Ministry of Education for Advanced Display and System

Application, Shanghai University, Shanghai 200072, China

Full list of author information is available at the end of the article
}

PART_nR $\times 2 \mathrm{~N}$, respectively. Among these eight labels, uppercase $\mathrm{N}$ denotes half the length of current CU's length or width and lowercase $\mathrm{n}$ signals one quarter of that [14]. It is akin to CU that PU brings the information about prediction. If current $\mathrm{CU}$ is encoded with intraprediction, it is always divided using square partition, namely INTRA_2N $\times 2 \mathrm{~N}$ and INTRA_N $\times \mathrm{N}$. As illustrated in Fig. 1, a CU can either be coded as a single PU or it can be split into two or four rectangular PUs.

After PU division, motion estimation (ME) is performed to find out the optimal motion vector (MV) of current block through matching corresponding samples. The computational load of ME in HEVC is related to the motion search range in matching process. For the full search range, 128 candidate points (configured as [- 64, 64]) are adopted in horizontal and vertical directions, respectively. In other words, more than 16,000 points should be searched to find the best MV for current block. Although the fast ME approach TZ Search is added into the latest HEVC reference software to simplify the MV search process, the coding complexity is still very high. In interprediction stage, asymmetric partition (AMP) partitioning and ME process can promote the matching precision. However, the coding time is increased significantly for the novel complex design. 
Therefore, this paper presents an efficient approach to reduce the calculation burden in interprediction. Firstly, we find out a fast decision method so that some prediction units are divided into asymmetric partitions directly without the complex calculation. And then, based on the motion degree function we defined, the motion search range of ME can be adaptively adjusted.

The remainder content of this article is organized as follows. Our proposed method is described in Section 2. Section 3 demonstrates the experimental comparison between our proposed approach and other methods and discusses the coding performance of the proposed algorithm. Finally, we summarize this paper in Section 4.

\subsection{The related previous work}

To reduce interprediction computational burden for HEVC encoder, many researchers have proposed various fast methods. These methods aim to simplify PU mode decision process that we sum up as follows. A different $\mathrm{CU}$ visiting order is stated in Ref. [15], and this modification reduces the complexity of $\mathrm{CU}$ size decision, mode decision, and interframe prediction. Another fast $\mathrm{CU}$ size prediction for HEVC is presented in Ref. [16], which early terminates partitioning modes to reduce computational complexity. Ref. [17] adopts convolution neural network to decrease no less than two CU partition modes for rate distortion optimization (RDO). A set of procedures for partition structure determination is designed in Ref. [18] to early terminate exhaustive RDO process using data mining techniques. An early terminating fast intermode decision algorithm, namely hierarchical intermode decision (HIMD), is presented in Ref. [19] using the correlation between RD cost and PU size. A course of low complexity mode decision algorithm consists of four early termination strategies and this approach uses co-located block depth information and current block size information to terminate RDO process [20]. A reverse $\mathrm{CU}$ access approach is proposed in Ref. [21] that can further reduce HEVC coding complexity, particularly for high motion activity sequences. A fast CU partitioning algorithm is provided in Ref. [22] for HEVC, and this method early terminates the $\mathrm{CU}$ size decision process based on the Bayesian decision rule. A fast interprediction mode decision algorithm is proposed in our previous work [23] that uses current block edge information to simplify RDO process so as to accelerate coding time for HEVC encoder.

Furthermore, complex ME process in HEVC encoder requires a huge calculation, so many low-complexity algorithms have been presented to make improvement on ME process. A block motion information derivation is described in Ref. [24] without conventional motion estimation and motion parameters. A fast algorithm is introduced in Ref. [25] to reduce the number of ME searching points from 81 to just 31. A fast ME decision method is provided in Ref. [26] that skips block search process adaptively on the basis of the relationship between the two types PU after classifying PUs into two classes. Two individual algorithms are proposed in Ref. [27] that obtain the best performance collaboratively. A better balance is stated between computational burden and RD performance in Ref. [28]. In Ref. [29], authors state fast mode decision algorithm not only for CU size decision but also for ME decision, and it achieves a great time saving.

Admittedly, all of these approaches have improved coding efficiency of intercoding for HEVC encoder. However, it is hard for interprediction optimization to balance the computational complexity and the coding performance. Motivated by the abovementioned issue, we propose an efficient AMP decision and search range adjustment algorithm for HEVC.

\subsection{Preliminary work}

Because of the huge amount of data, especially when having an advanced hierarchical partition design in current HEVC standard, compression of high-definition video content relies heavily on the efficient compression of video information. Note that, one way of performing prediction mode decision would exhaustively search the possibilities within a set containing all possible modes, which results in an undesirable complexity. To determinate the optimal mode, it adopts Lagrange multiplier to find the certain mode with the least RD cost as the best one, and the RD cost function $(J)$ is shown as follows:

$$
J=D_{\text {mode }}+\lambda \times B R_{\text {mode }}
$$

where $D_{\text {mode }}$ denotes the distortion between the current mode and the matching mode on average, $\lambda$ signifies the Lagrange multiplier, and $B R_{\text {mode }}$ is bits required for signaling the coding mode and the associated side information. Moreover, CU division way in HEVC is identical to that way in H.264/AVC, while new PU partitions (including square partition (SP), symmetric partition (SMP), and AMP) have been developed for HEVC interprediction. In contrast to H.264/AVC intermode prediction, before the decision of the optimal MV for a block, all the SP, SMP, and AMP in PU modes are added to the pre-decision list. And then, all possible search point candidates corresponding to the PU mode in predecision list are considered as full RD search list to perform ME using full search range. During this procedure, the distortion among all candidates (including PU partitions and search points) is computed and the best prediction mode is selected. This technique enhances the highest possible coding precision in HEVC encoder, but the "try all and select the best" method leads to 


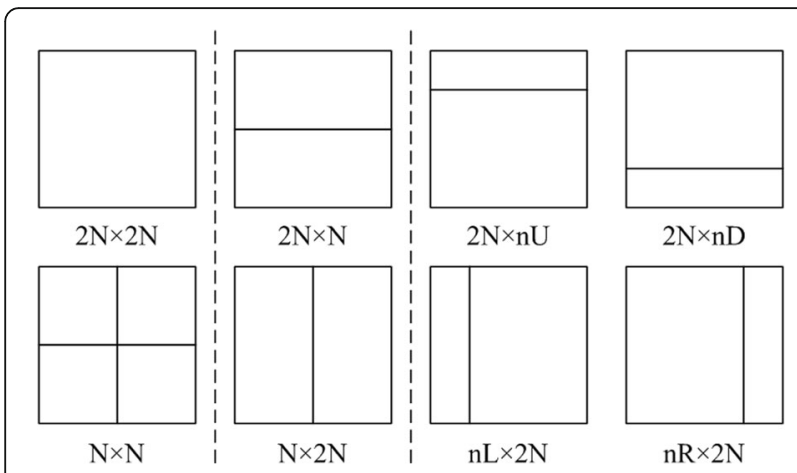

Fig. 1 The eight PU partitions. Left: square partition (SP); middle: symmetric partition (SMP); right: asymmetric partition (AMP)

extremely high coding complexity, so it limits HEVC for practical applications.

In preliminary work, prediction modes distribution has been checked at RA (RandomAccess configuration) and LDP (Lowdelay $\mathrm{P}$ configuration) conditions. Figure 2 shows the average area shares of prediction modes in HEVC encoder at RA and LDP conditions. It is obvious that with the reduction of quantization parameter $(\mathrm{QP})$, more and more blocks choose AMP as the best interprediction mode under both RA and LDP configurations. Although the share of AMP is lower than other mode decision, it is very time-consuming to perform AMP for interprediction modes. Furthermore, rather than intracoding, all of interprediction modes need to be addressed with ME, which results in both high calculation burden and a long coding time. To analyze the computational load of AMP partitioning and ME process in HEVC encoder, extensive experiments were conducted on ten text sequences with QP setting as 22, 27, 32, and 37 . These settings are tabulated in Table 1 according to common test condition (CTC) [30], and HEVC test model 14.0 (HM $14.0)$ is used as the experimental platform.

The encoder configuration is as follows: GOP length is set to 8 with an intraperiod being 32; the maximum $\mathrm{CU}$ size is $64 \times 64$, and $\mathrm{CU}$ depth level is set to 4 ; search range of $\mathrm{ME}$ is configured as [-64,64]. We execute simulated experiments twice with AMP disabled (off) and enabled (on) for each test sequence, and the original HM reference software (AMP on) is regarded as the benchmark. Moreover, computational load for these experiments is reflected by coding time which is calculated by $\Delta T=\frac{T_{\mathrm{off}}^{\text {avg }}-T_{\mathrm{on}}^{\text {avg }}}{T_{\text {on }}^{\text {avg }}}$, where $T_{\text {off }}^{\text {avg }}$ and $T_{\text {on }}^{\text {avg }}$ mean the average coding time when AMP off and AMP on, respectively. In addition, peak signal-to-noise ratio (PSNR) change, bitrate variation, and coding time change are measured by "BD-PSNR (dB)" (Bjontegaard Delta PSNR), "BD-BR (\%)" (Bjontegaard Delta Bitrate), and " $\Delta T(\%)$ " [31].

Table 2 illustrates the coding efficiency comparison between the HM 14.0 with AMP off and the original HM 14.0 with AMP on both under RA and LDP cases. The minus and the positive number respectively denote drop and augment compared with the data derived by the benchmark (the same as in the following tables). From the experimental results, one can notice from Table 1 that the maximum time saving of $18.12 \%$ for "BasketballDrive" under LDP configuration when AMP is disabled. The coding time is averagely reduced about 12.83 and $14.05 \%$ under "RA" and "LDP" case, respectively. It can be seen that disabling AMP can speed up encoding process. While for the sequences with small movement activity, such as "BQSquare" and "Johnny", PSNR is close to that of original HEVC encoder with AMP off. However, when AMP is disabled, both PSNR loss and bitrate increase are obviously significant. It also can be seen from Table 2 that for test sequences with much high motion activity areas, such as "BasketballDrive," "BasketballDrill," and "RaceHorses," the

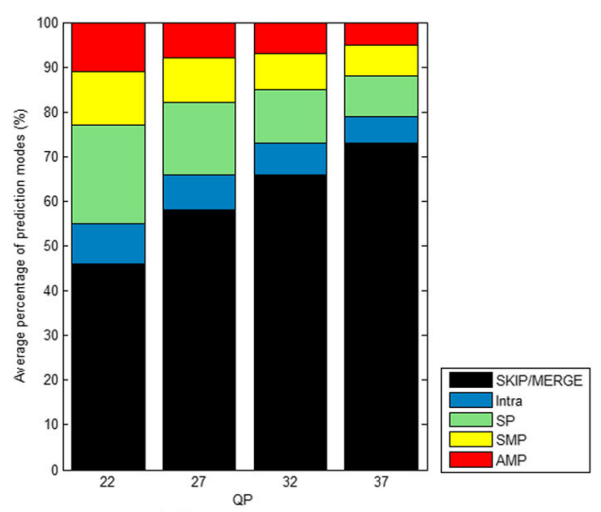

(a) at R_A condition

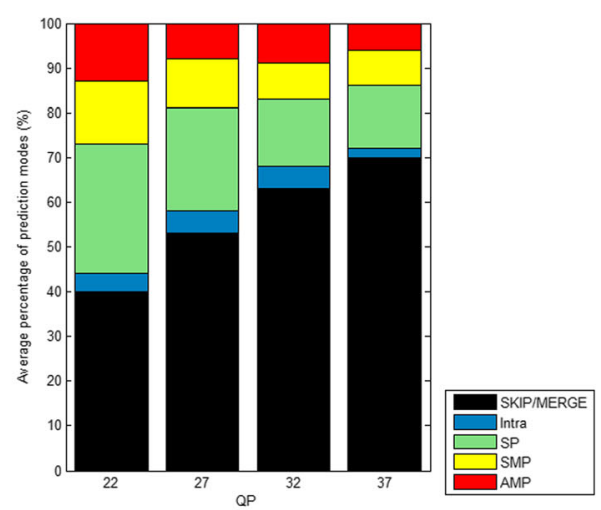

(b) at L_P condition

Fig. 2 Prediction mode distribution. a At R_A condition. $\mathbf{b}$ At L_P condition 
Table 1 Test sequence information

\begin{tabular}{llllll}
\hline Class & Resolution & Test sequence & Frame rate & Bit depth & $\begin{array}{l}\text { Motion } \\
\text { degree }\end{array}$ \\
\hline A & $2560 \times 1600$ & Traffic & $30 \mathrm{fps}$ & 8 & High \\
& & PeopleOnStreet & $30 \mathrm{fps}$ & 8 & Low \\
B & \multirow{2}{*}{$1920 \times 1080$} & BasketballDrive & $50 \mathrm{fps}$ & 8 & High \\
& & Cactus & $50 \mathrm{fps}$ & 8 & Low \\
C & \multirow{2}{*}{$832 \times 480$} & BQMall & $60 \mathrm{fps}$ & 8 & Low \\
& & BasketballDrill & $50 \mathrm{fps}$ & 8 & High \\
D & \multirow{2}{*}{$416 \times 240$} & BQSquare & $60 \mathrm{fps}$ & 8 & Low \\
& & RaceHorses & $30 \mathrm{fps}$ & 8 & High \\
E & \multirow{2}{*}{$1280 \times 720$} & FourPeople & $60 \mathrm{fps}$ & 8 & Low \\
& & Johnny & $60 \mathrm{fps}$ & 8 & Low \\
\hline
\end{tabular}

coding time reduction for them is relatively more than that of other test sequences after disabling AMP. Because turning off AMP can eliminate amounts of complicated ME process, computational load is saved conspicuously, and yet PSNR loss and bitrate increase are evident.

These results demonstrate that there is much room to simplify the interdecision process in HEVC. Accordingly, if we can exploit certain information to adaptively stop some specific blocks executing AMP prediction, and if the complex ME process can be simplified in HEVC encoder, the calculation load will be reduced dramatically in the whole HEVC interencoding process.

\section{The proposed efficient method for HEVC intercoding}

As presented in the previous sections, the complicated intermode decision consumes plenty of coding time, which prevents HEVC encoder from practical application. Hence, optimizing interprediction is a main challenge for fulfilling coding time saving.

\subsection{AMP decision skipping algorithm}

Through analyzing comparative results in Section 1, notwithstanding disabling AMP can avoid complicated ME process, most of CUs are further split. Thus, further splitting damages PSNR and bitrates simultaneously. For current CU, more coding time will be saved if the optimal prediction mode is determined in advance. We would like to propose an efficient AMP decision algorithm that can early decide whether AMP is the best partition or not.

According to the simulated results in Table 2, we compare sequence content between AMP on and AMP off. The test sequence "RaceHorses" is taken as an example. We can observe from Fig. 3 that when AMP is disabled, just a small part of CUs carry out SKIP mode (in blue rectangle). While some CUs are partitioned by AMP when AMP is enabled, but their corresponding CUs were split into smaller sub-CUs (in red rectangle) when AMP is disabled. This is the reason that coding time reduces not sufficiently while the loss of coding performance is not so much when AMP is disabled.

As mentioned by [32-34], it is just an MV that distinguishes the current $\mathrm{CU}$ and its co-located $\mathrm{CU}$ in previous coded frame. Accordingly, the relationship between them can be modeled as $I(p+m v, t-1)=I(p, t)$, where $I(p, t)$ denotes the value of pixel in $p$ position at $t$ time and $m v$ represents motion vector. Furthermore, the high correlation between $\mathrm{CU}$ and its co-located $\mathrm{CU}$ has been verified in $[33,34]$. In accordance with this high correlation, we conduct extensive experiments on ten sequences in Table 1 to discover the relationship between AMP and $\mathrm{CU}$ position. As illustrated in Fig. 4, current $\mathrm{CU}$ is termed as $C U_{\text {cur }}$, which should have been encoded as AMP with AMP off. Its corresponding $\mathrm{CU}$ is labeled as $C U_{\text {cor }}$ which is decided as AMP. In addition, the co-located $\mathrm{CU}$ of $C U_{\text {cor }}$ is labeled as $C U_{\text {col }}$. The

Table 2 Comparison between HM 14.0 (AMP off) and the original HM 14.0 (AMP on)

\begin{tabular}{|c|c|c|c|c|c|c|}
\hline \multirow[t]{2}{*}{ Test sequence } & \multicolumn{3}{|l|}{ RA } & \multicolumn{3}{|l|}{ LDP } \\
\hline & BD-PSNR (dB) & BD-BR (\%) & $\Delta T(\%)$ & BD-PSNR (dB) & BD-BR (\%) & $\Delta T(\%)$ \\
\hline Traffic & -0.03 & 0.78 & -10.45 & N/A & N/A & N/A \\
\hline PeopleOnStreet & -0.04 & 0.67 & -9.57 & N/A & N/A & N/A \\
\hline BasketballDrive & -0.06 & 1.16 & -16.31 & -0.11 & 1.21 & -18.12 \\
\hline Cactus & -0.05 & 0.98 & -11.06 & -0.06 & 0.87 & -14.97 \\
\hline BQMall & -0.05 & 1.22 & -13.84 & -0.07 & 0.93 & -12.36 \\
\hline BasketballDrill & -0.06 & 1.17 & -15.63 & -0.09 & 1.08 & -17.21 \\
\hline BQSquare & -0.02 & 0.54 & -7.82 & -0.05 & 0.86 & -15.39 \\
\hline RaceHorses & -0.06 & 1.24 & -17.94 & -0.03 & 0.77 & -13.58 \\
\hline FourPeople & N/A & N/A & N/A & -0.04 & 0.82 & -11.32 \\
\hline Johnny & N/A & N/A & N/A & -0.02 & 1.13 & -9.42 \\
\hline Average & -0.05 & 0.97 & -12.83 & -0.06 & 0.96 & -14.05 \\
\hline
\end{tabular}




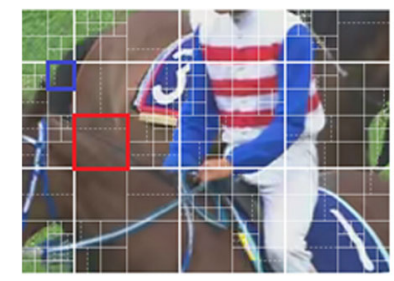

(a) AMP on

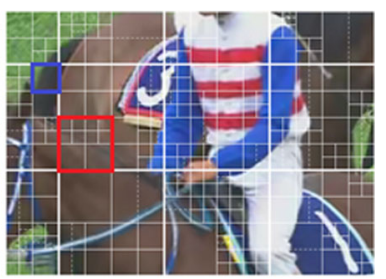

(b) AMP off
Fig. $3 \mathrm{CU}$ and PU partition of the second frame in "RaceHorses" under RA case with QP of 32. a AMP on. $\mathbf{b}$ AMP off

experiments are to calculate the probabilities of mode distribution of $C U_{\text {col }}$ and $C U_{\text {cor }}$.

Table 3 shows the posterior probabilities of $C U_{\text {cur }}$ mode selection and $C U_{\text {col }}$ mode selection, where $M_{\text {cur }}$ and $M_{\text {col }}$ represent the optimal mode of $C U_{\text {cur }}$ and $C U_{\text {col }}$, respectively. It is obvious from Table 3 that if $C U_{\text {cur }}$ are selected as SKIP mode with AMP disabled, over $96 \%$ of $C U_{\text {col }}$ in previous coded frame is decided as SKIP mode when AMP is enabled. In particular, when QP selects 37, the probability of SKIP mode for $C U_{\text {col }}$ reaches up to $98.7 \%$. It demonstrates that if $M_{\text {cur }}$ chooses SKIP mode, $M_{\text {col }}$ will also be SKIP mode likely. However, if $C U_{\text {cur }}$ is further split when AMP is off, the modes on $C U_{\text {col }}$ distribute evenly $(20.4 \%$ of SKIP mode, $18.8 \%$ of MERGE mode, $53.1 \%$ of three partition modes and $7.7 \%$ of intramode). Because of the high relevance between two adjacent frames, AMP decision on current $\mathrm{CU}$ can be avoided adaptively based on whether $M_{\mathrm{col}}$ is SKIP mode or not. In other words, AMP decision on one CU should be skipped immediately without any RD cost calculation process if the co-located $\mathrm{CU}$ in previous coded frame of this CU was encoded as SKIP mode. Otherwise, current $\mathrm{CU}$ will be predicted as AMP or still as SMP. This approach achieves some AMP decision skipping with sacrificing coding quality, while this loss is

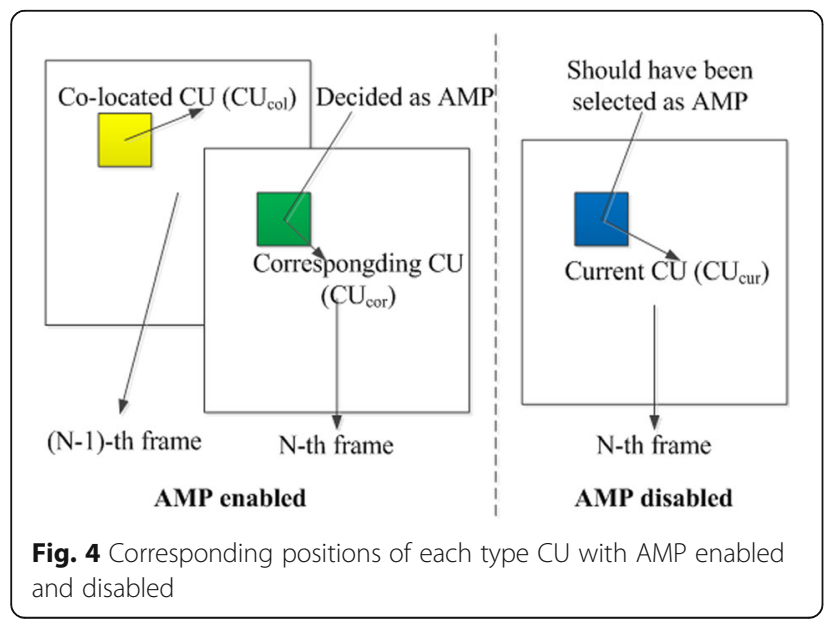

Table 3 Posterior probabilities of $C U_{\text {cur }}$ mode selection and $\mathrm{CU}_{\text {col }}$ mode selection

\begin{tabular}{|c|c|c|c|c|c|c|}
\hline \multirow[t]{2}{*}{$M_{\text {cur }}$} & \multirow[t]{2}{*}{$M_{\text {col }}$} & \multicolumn{4}{|c|}{ Probability $\left(P\left(M_{\text {col }} \mid M_{\text {cur }}\right), \%\right)$} & \multirow[t]{2}{*}{ Average } \\
\hline & & $\mathrm{QP}=22$ & $\mathrm{QP}=27$ & $\mathrm{QP}=32$ & $Q P=37$ & \\
\hline \multirow[t]{6}{*}{ SKIP } & SKIP & 94.2 & 95.5 & 96.3 & 98.7 & 96.2 \\
\hline & MERGE & 0.8 & 0.6 & 0.5 & 0.1 & 0.5 \\
\hline & SP & 1.1 & 1.0 & 0.8 & 0.4 & 0.9 \\
\hline & SMP & 1.7 & 1.4 & 1.2 & 0.5 & 1.2 \\
\hline & AMP & 1.6 & 1.3 & 0.9 & 0.3 & 1.0 \\
\hline & Intra & 0.6 & 0.2 & 0.3 & 0.0 & 0.3 \\
\hline \multirow[t]{6}{*}{ Further split } & SKIP & 18.5 & 20.1 & 21.3 & 21.8 & 20.4 \\
\hline & MERGE & 18.3 & 19.7 & 18.1 & 18.9 & 18.8 \\
\hline & SP & 19.2 & 20.9 & 22.1 & 20.0 & 20.6 \\
\hline & SMP & 19.3 & 18.4 & 17.9 & 16.5 & 18.0 \\
\hline & AMP & 13.4 & 14.8 & 14.2 & 15.7 & 14.5 \\
\hline & Intra & 11.3 & 6.1 & 6.4 & 7.1 & 7.7 \\
\hline
\end{tabular}

quite negligible and the computational load reduction is very significant.

\subsection{Search range adjustment algorithm}

$\mathrm{ME}$ is the most computationally expensive procedure in HEVC encoder. The search range of the matched sample restrains current block to obtain the best MV within a limited area in the reference frame. Larger the search range is, higher the computational burden costs. Contrarily, a very small motion search range contains a few candidate points for matching so that the best $\mathrm{MV}$ can be checked out within a short time. An appropriate search range is capable of saving considerable search time and at the same time keeping a good RD performance for HEVC encoder. However, due to small size search range has poor candidates, a low precision and even an extreme error will be generated. In addition, a large motion search range is indispensable for reliable prediction. Therefore, the motion search range can be adjusted properly without loss of HEVC coding efficiency.

In this section, the coding information (including search range and $\mathrm{CU}$ size) from the spatial adjacent blocks will be exploited to discover the characteristics among spatial blocks in the same frame and to determine search range for current CU. Extensive experiments were conducted on six typical text sequences from Table 1 , and simulated conditions are same as configuration in Section 1.

Six selected test sequences in Table 4 can be encoded with both RA and LDP cases according to CTC. Sequences "BasketballDrive," "BasketballDrill," and "RaceHorses" have many fast moving objects, while sequences "Cactus," "BQMall," and "BQSquare" are with relatively low movement activity, and "BQSquare" even has large static background. For better understanding 
Table 4 Distribution of CU sizes for six typical test sequences

\begin{tabular}{|c|c|c|c|c|c|c|c|c|c|}
\hline \multirow[t]{3}{*}{ Sequences } & \multirow[t]{3}{*}{ QP } & \multicolumn{8}{|c|}{ CU size distribution (area normalization) } \\
\hline & & \multicolumn{4}{|c|}{ RA } & \multicolumn{4}{|l|}{ LDP } \\
\hline & & $64 \times 64$ & $32 \times 32$ & $16 \times 16$ & $8 \times 8$ & $64 \times 64$ & $32 \times 32$ & $16 \times 16$ & $8 \times 8$ \\
\hline \multirow[t]{4}{*}{ BasketballDrive } & 22 & 0.03 & 0.08 & 0.23 & 0.66 & 0.03 & 0.10 & 0.22 & 0.64 \\
\hline & 27 & 0.04 & 0.10 & 0.26 & 0.61 & 0.04 & 0.11 & 0.24 & 0.61 \\
\hline & 32 & 0.05 & 0.09 & 0.28 & 0.58 & 0.05 & 0.12 & 0.24 & 0.59 \\
\hline & 37 & 0.06 & 0.11 & 0.28 & 0.55 & 0.05 & 0.13 & 0.21 & 0.61 \\
\hline \multirow[t]{4}{*}{ BasketballDrill } & 22 & 0.03 & 0.08 & 0.20 & 0.69 & 0.03 & 0.09 & 0.25 & 0.63 \\
\hline & 27 & 0.04 & 0.10 & 0.24 & 0.63 & 0.04 & 0.10 & 0.26 & 0.61 \\
\hline & 32 & 0.04 & 0.10 & 0.25 & 0.61 & 0.05 & 0.11 & 0.25 & 0.59 \\
\hline & 37 & 0.04 & 0.10 & 0.23 & 0.63 & 0.05 & 0.11 & 0.25 & 0.59 \\
\hline \multirow[t]{4}{*}{ RaceHorses } & 22 & 0.02 & 0.07 & 0.29 & 0.62 & 0.02 & 0.13 & 0.27 & 0.58 \\
\hline & 27 & 0.03 & 0.08 & 0.30 & 0.59 & 0.04 & 0.14 & 0.31 & 0.52 \\
\hline & 32 & 0.03 & 0.08 & 0.34 & 0.56 & 0.03 & 0.16 & 0.32 & 0.49 \\
\hline & 37 & 0.04 & 0.09 & 0.31 & 0.56 & 0.03 & 0.16 & 0.31 & 0.51 \\
\hline Average & & 0.04 & 0.09 & 0.27 & 0.61 & 0.04 & 0.12 & 0.26 & 0.58 \\
\hline \multirow[t]{4}{*}{ Cactus } & 22 & 0.32 & 0.52 & 0.13 & 0.04 & 0.35 & 0.47 & 0.11 & 0.07 \\
\hline & 27 & 0.33 & 0.48 & 0.14 & 0.04 & 0.37 & 0.48 & 0.11 & 0.05 \\
\hline & 32 & 0.33 & 0.50 & 0.13 & 0.04 & 0.37 & 0.48 & 0.11 & 0.04 \\
\hline & 37 & 0.35 & 0.48 & 0.14 & 0.03 & 0.38 & 0.47 & 0.12 & 0.03 \\
\hline \multirow[t]{4}{*}{ BQMall } & 22 & 0.34 & 0.51 & 0.11 & 0.04 & 0.40 & 0.45 & 0.11 & 0.04 \\
\hline & 27 & 0.37 & 0.50 & 0.10 & 0.03 & 0.41 & 0.44 & 0.11 & 0.05 \\
\hline & 32 & 0.36 & 0.52 & 0.09 & 0.04 & 0.42 & 0.43 & 0.11 & 0.04 \\
\hline & 37 & 0.38 & 0.48 & 0.09 & 0.04 & 0.41 & 0.45 & 0.10 & 0.04 \\
\hline \multirow[t]{4}{*}{ BQSquare } & 22 & 0.48 & 0.40 & 0.08 & 0.03 & 0.50 & 0.40 & 0.07 & 0.03 \\
\hline & 27 & 0.44 & 0.44 & 0.09 & 0.03 & 0.52 & 0.39 & 0.06 & 0.04 \\
\hline & 32 & 0.45 & 0.46 & 0.07 & 0.01 & 0.48 & 0.43 & 0.06 & 0.03 \\
\hline & 37 & 0.46 & 0.42 & 0.10 & 0.01 & 0.53 & 0.38 & 0.06 & 0.03 \\
\hline Average & & 0.38 & 0.48 & 0.11 & 0.03 & 0.43 & 0.44 & 0.09 & 0.04 \\
\hline
\end{tabular}

for readers, based on the average portions of each $\mathrm{CU}$ size, we normalize the distribution data using area normalization method. The area normalization method can be used to measure the fineness of specimen in chemistry, while we adopt this method to further highlight the $\mathrm{CU}$ size distribution in this paper. For the sequences with complex motion objects ("BasketballDrive," "BasketballDrill," and "RaceHorses"), their small size CU $(16 \times 16$ and $8 \times 8)$ is close to 0.90 under RA configuration, as well as 0.84 under LDP configuration as shown in Table 4. In particular, as a test sequence with large global motion area, sequence "RaceHorses" has a huge amount of movement information, so the small size $\mathrm{CU}$ in this sequence also accounts for nearly 0.90 at RA condition. It is because that motion in these test sequences is quite rich and objects in them move dramatically, it needs small size CU to record abundant movement information for fulfilling an accurate MV coding. Nevertheless, in the other three test sequences with comparative simple motion, the overwhelming majority of CU is big size $(64 \times 64$ and $32 \times 32)$, where it is from 0.83 ("Cactus" in RA case) to 0.90 ("BQSquare" in LDP case) on average at both two conditions. Particularly "BQSquare" has large motionless region, so CUs in this test sequence are almost totally chosen as big size. Another observation from Table 4 is that as QP value is rising, the share of big size $\mathrm{CU}$ is growing, since growth of QP smoothens the video texture so that the motion degree is slow. The region in current $\mathrm{CU}$ with big size is covered by homogeneous texture or motion content, while the small size CUs carry a large number of texture and movement information. As a result, we can initially determine motion degree upon the current CU size.

Based on aforementioned observations, we can find that motion degree is relative closely to $\mathrm{CU}$ size. Moreover, the first three sequences contain complex motion, so the $\mathrm{CU}$ size in these four sequences always tends to 
be small. While the motion in the last three sequences is relatively slow, therefore, the big $\mathrm{CU}$ accounts for the vast majority. In other words, fast/slow motion leads to small/big size $\mathrm{CU}$ and requires big/small motion search range. Consequently, according to the motion information of CU size, various $\mathrm{CU}$ sizes are divided into four categories and the weight value $(\omega)$ can be setup on different CU sizes, as shown in Table 5.

MVs of the current $\mathrm{CU}$ are always correlated with the MVs of its spatially neighboring blocks [33]. It is possible that the current block and its spatially adjacent blocks have the same moving object with similar movement information and the motion of the object is unlikely to alter abruptly over time. Accordingly, the relationship among movement information of the spatially neighboring blocks can be utilized to adjust search range for current CU. Furthermore, a plenty of experiments have been conducted on various test sequences, and it is observed that most objects move along horizontal or vertical direction. Hence, we tabulate Table 6 to assign the relationship factor $\left(\beta_{i}\right)$ between current $\mathrm{CU}$ and its adjacent spatial blocks. The spatial correlation is one of the major parameters for measuring motion degree of current CU [35]. Additionally, another crucial parameter to judge the degree is current CU size, where different sizes are assigned different weight values as tabulated in Table 5. Therefore, we can determine the motion degree of current $\mathrm{CU}$ in accordance with the weight value $(\omega)$ and the relationship factors $\left(\beta_{i}\right)$ as follows:

$$
M D=\omega \sum_{i=0}^{3} \omega_{i} \cdot \beta_{i} \cdot f_{i}
$$

where $M D$ represents motion degree of current $\mathrm{CU}$, and weight value $\omega_{i}$ is signified by search ranges of neighboring block $i$ according to results in Table 5, as well as the flag $f_{i}$ is set as 1 if neighboring block $i$ is available; otherwise, it is assigned as 0 .

In general, the larger the parameter $M D$ is, the stronger the uncertainty of motion is and the bigger the search range is. According to this regulation, the search range of current $\mathrm{CU}$ is able to be determined through Table 7. $T_{1}, T_{2}$, and $T_{3}$ in Table 7 are thresholds to classify current $\mathrm{CU}$ into each types. Inspired by [35] and through a great deal of experimental

Table 5 Weight values for each CU size

\begin{tabular}{ll}
\hline CU size & Weight value $(\omega)$ \\
\hline $64 \times 64$ & 0.1 \\
$32 \times 32$ & 0.2 \\
$16 \times 16$ & 0.3 \\
$8 \times 8$ & 0.4 \\
\hline
\end{tabular}

Table 6 Relationship factor $\left(\beta_{i}\right)$ and position of neighboring block

\begin{tabular}{lll}
\hline$i$ & Relationship factor $\left(\beta_{i}\right)$ & Position of neighboring block \\
\hline 0 & 4 & Left block \\
1 & 1 & Top-left block \\
2 & 4 & Top block \\
3 & 1 & Top-right block \\
\hline
\end{tabular}

results, we achieve the accuracies of the proposed algorithm using various thresholds, which are shown in Table 8. The accuracies here are defined as the matching rate between the MVs by the proposed algorithm and the MVs by the original HEVC encoder. $T_{1}, T_{2}$, and $T_{3}$ are assigned to $0.65,10.50$, and 13.00 , respectively. This configuration is capable of adjusting search range for current $\mathrm{CU}$, which avoids extremely high computational load from full search range and achieves a consistent coding performance.

\subsection{Overall algorithms}

On the basis of the aforementioned analysis, our proposed efficient approach for HEVC combines fast PU partition decision algorithm and fast ME process algorithm. The flow chart of our proposed overall algorithms is illustrated as Fig. 5.

We further describe the steps in detail as follows:

Step 1: Start interprediction for a CU.

Step 2: Determine whether current $\mathrm{CU}$ is partitioned to SMP (PART_2N $\times \mathrm{N}$ and PART_N $\times 2 \mathrm{~N}$ ) or not. If current $\mathrm{CU}$ is divided into SMP, go to step 3; otherwise, go to step 4.

Step 3: Perform AMP decision skipping. Derive the co-located CU in previous coded frame of current CU; if it was encoded as SKIP mode, current $\mathrm{CU}$ should also be decided as SKIP mode and go to step 6; otherwise, current CU should further be decided as AMP or still as SMP and go to step 4.

Step 4: Perform fast search range adjustment.

Step 4.1: Set the weight value $(\omega)$ upon each CU sizes and assign each search ranges corresponding to different $\omega$ as shown in Table 5 .

Step 4.2: Compute motion degree of current CU based on Eq. (2) using several parameters, including relationship factor from Table 6.

Table 7 Search range adjustment using MD

\begin{tabular}{ll}
\hline Search range & Condition \\
\hline$[-8,8]$ & $M D<T_{1}$ \\
{$[-16,16]$} & $T_{1} \leq M D<T_{2}$ \\
{$[-32,32]$} & $T_{2} \leq M D<T_{3}$ \\
{$[-64,64]$} & $T_{3} \leq M D$ \\
\hline
\end{tabular}


Table 8 Comparison between ADS and the original HM 14.0

\begin{tabular}{|c|c|c|c|c|c|c|}
\hline \multirow[t]{2}{*}{ Test sequence } & \multicolumn{3}{|l|}{ RA } & \multicolumn{3}{|l|}{ LDP } \\
\hline & BD-PSNR (dB) & BD-BR (\%) & $\Delta T(\%)$ & BD-PSNR (dB) & BD-BR (\%) & $\Delta T(\%)$ \\
\hline Traffic & -0.03 & 0.47 & -8.26 & $\mathrm{~N} / \mathrm{A}$ & N/A & $\mathrm{N} / \mathrm{A}$ \\
\hline PeopleOnStreet & -0.03 & 0.51 & -8.38 & N/A & N/A & N/A \\
\hline BasketballDrive & -0.03 & 0.73 & -13.84 & -0.08 & 0.91 & -14.33 \\
\hline Cactus & -0.05 & 0.71 & -9.93 & -0.05 & 0.68 & -12.18 \\
\hline BQMall & -0.04 & 0.82 & -10.41 & -0.06 & 0.72 & -10.07 \\
\hline BasketballDrill & -0.02 & 0.87 & -12.55 & -0.06 & 0.85 & -13.46 \\
\hline BQSquare & -0.02 & 0.42 & -5.97 & -0.05 & 0.71 & -11.25 \\
\hline RaceHorses & -0.04 & 0.69 & -14.02 & -0.02 & 0.57 & -12.53 \\
\hline FourPeople & N/A & N/A & N/A & -0.03 & 0.63 & -9.76 \\
\hline Johnny & N/A & N/A & N/A & -0.02 & 0.89 & -8.87 \\
\hline Average & -0.03 & 0.65 & -10.42 & -0.05 & 0.75 & -11.56 \\
\hline
\end{tabular}

N/A not applicable

Step 4.3: According to values of $T_{1}, T_{2}$, and $T_{3}$, decide which search range for the current $\mathrm{CU}$ should be selected to execute ME.

Step 5: Perform ME with modified search range for current CU.

Step 6: Go to step 1 and address the next CU.

\section{Results and discussion}

This section analyzes the performance in terms of coding time, bitrates, and PSNR. In the following, the two sub-methods are described as (i) AMP decision skipping algorithm (labeled "ADS") and (ii) search range adjustment algorithm 2 (labeled "SRA").

For both algorithms, the HEVC test model (HM) reference software HM14.0 has been utilized. For these tests, ten sequences are released by JCT-VC group and the detailed information of the test sequences is provided in Table 1 in Section 1. It is noteworthy that all the ten test sequences in Table 1 are selected from the CTC and they have all kinds of resolutions (from $416 \times$ 240 to $2560 \times 1600)$ and various motion characteristics (low and high motion degree). All the experiments are

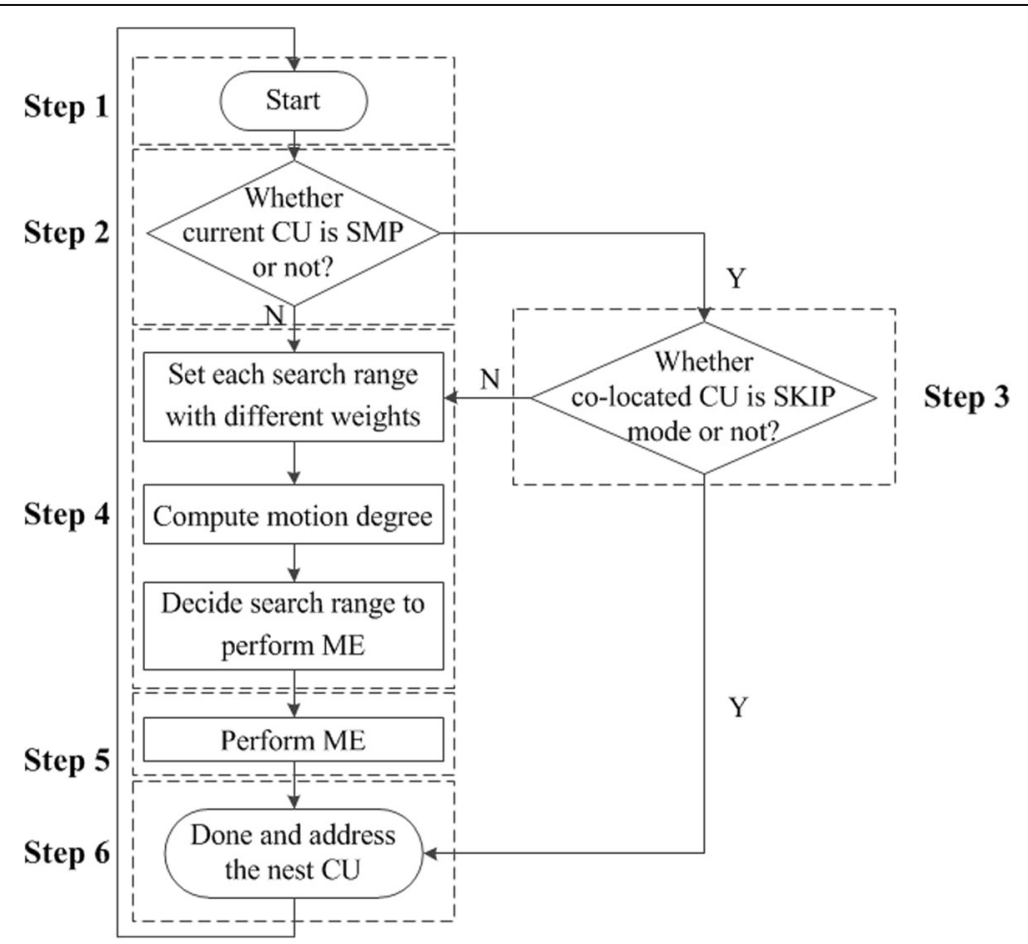

Fig. 5 Flow chart of our proposed overall algorithms 


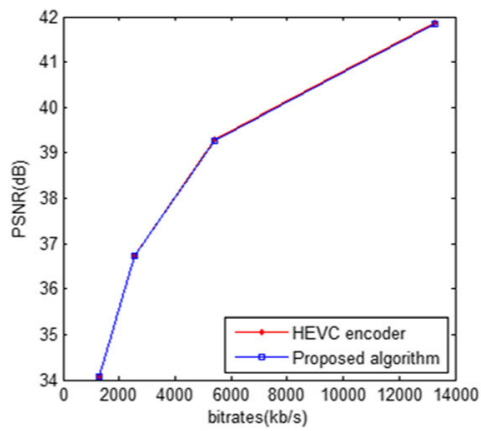

(a) "Traffic"

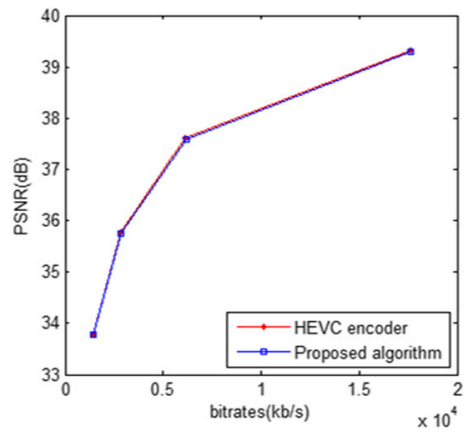

(c) "BasketballDrive"

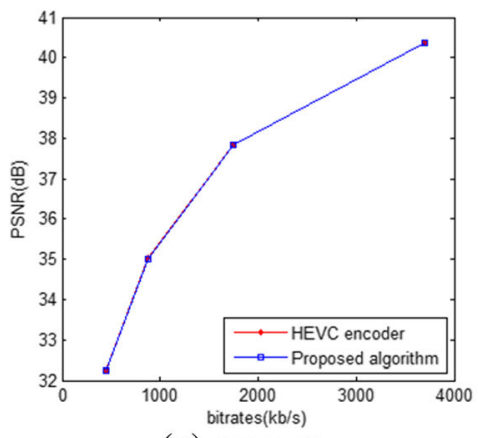

(e) "BQMall"

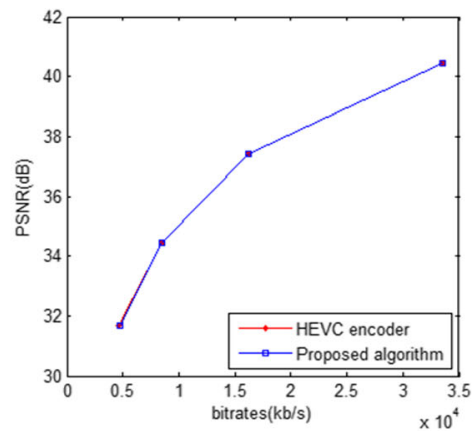

(b) "PeopleOnStreet"

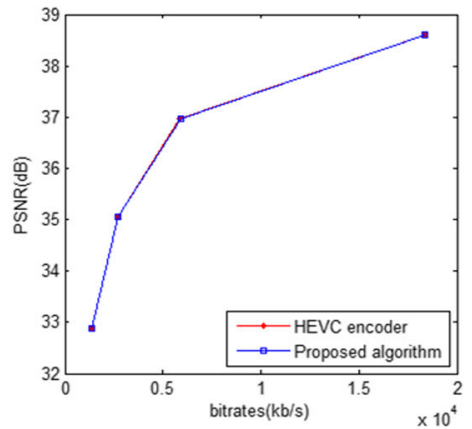

(d) "Cactus"

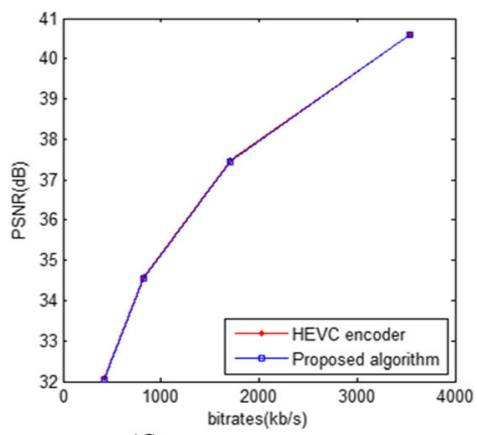

(f) "BasketballDrill"

Fig. 6 RD curves with the QP of 22, 27, 32 and 37 under RA configuration. a "Traffic". b "PeopleOnStreet". c "BasketballDrive". d "Cactus". e "BQMall". f "BasketballDrill"

simulated on two Intel Xeon E5-2640 v2 2.0GHz, 32GB DDR3 random access memory, and compiled on Microsoft Visual $\mathrm{C}++2010$ under RA and LDP cases. In addition, operating system is 64-bit Microsoft Windows 7 SP1. The encoder is configured as follows: both maximum CU width and height are 64 pixels, and maximum partition depth is 4 , resulting in minimum $\mathrm{CU}$ size of $8 \times 8$ pixels; period of I-Frame is 32 , and GOP (group of picture) size is 8; full search range of ME is set with 64; QP is respectively selected with $22,27,32$, and 37 ; RDO, SAO (sample adaptive offset), and AMP are enabled; and CABAC (context-based adaptive binary arithmetic coding) is used as the entropy coder.

In this section, intuitive comparison is shown in Figs. 6, 7, 8, and 9. Meanwhile, the results of comparative experiments are tabulated in Tables 8, 9, and 10 which show the results between our approach and the original HEVC encoder. Figures 10, 11, and 12 provide the results compared with the state-of-the-art fast intercoding methods in detail. Note that the same as Section 1, changes of PSNR, bitrates, and coding time are labeled by "BD-PSNR (dB)," "BD-BR (\%)," and “ $\Delta T(\%)$, " respectively.

\subsection{Evaluation of individual algorithm ADS compared with HM 14.0}

To assess the coding performance of our proposed ADS algorithm compared with the original HEVC encoder, we have conducted a large number of experiments, and the experimental results in Table 8 illustrate the relative encoding performance of the proposed ADS algorithm 


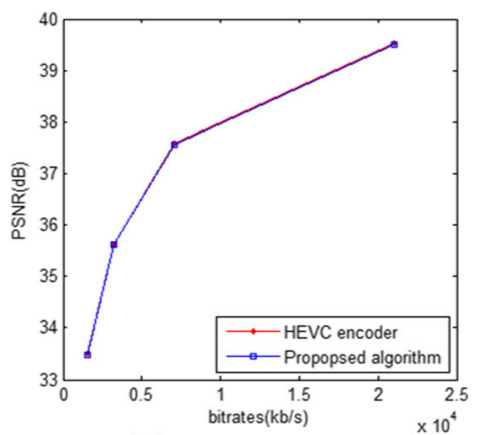

(a) "BasketballDrive"

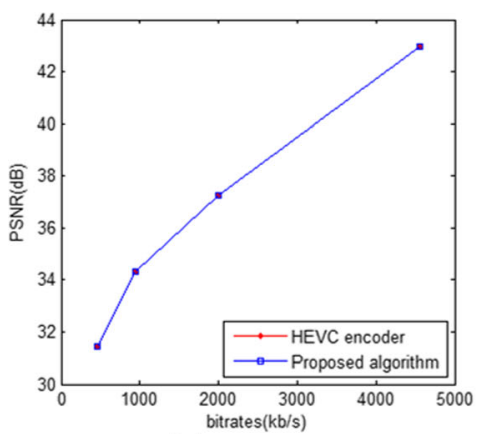

(c) "BQMall"

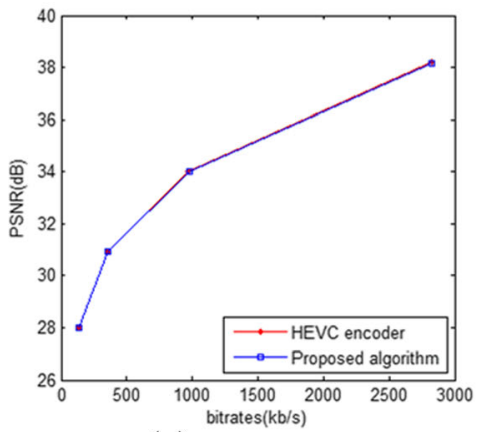

(e) "BQSquare"

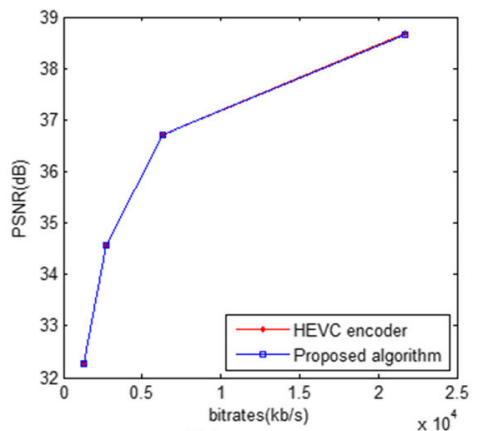

(b) "Cactus"

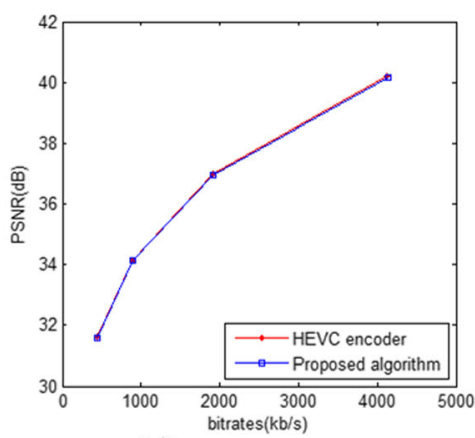

(d) "BasketballDrill"

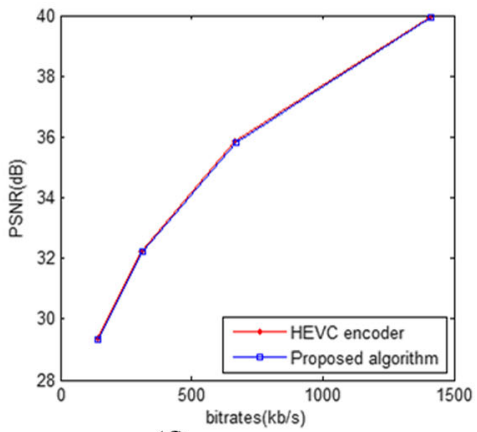

(f) "RaceHorses"

Fig. 7 RD curves with the QP of 22, 27, 32 and 37 under LDP configuration. a "BasketballDrive". b "Cactus". c "BQMall". d "BasketballDrill". e "BQSquare". f "RaceHorses"

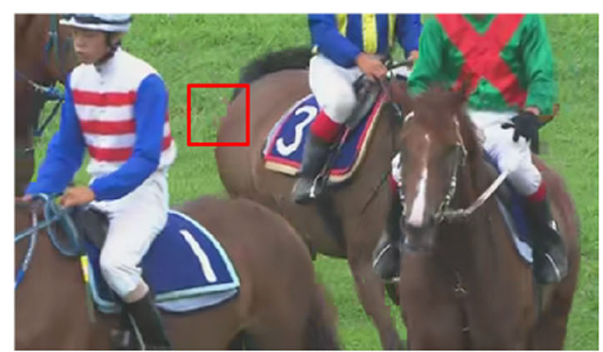

(a) encoded by the original HM 14.0

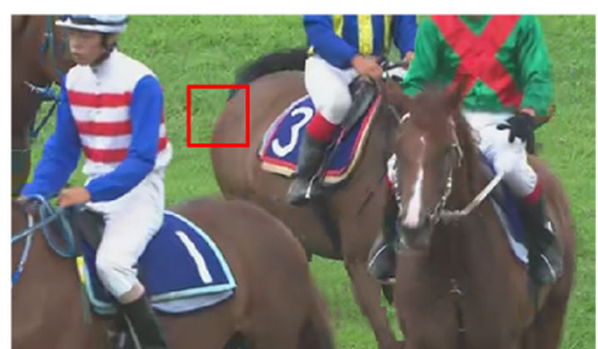

(b) encoded by the proposed algorithm

Fig. 8 Comparison of the subjective quality of the 22nd frame in "RaceHorses". a Encoded by the original HM 14.0. b Encoded by the proposed algorithm 


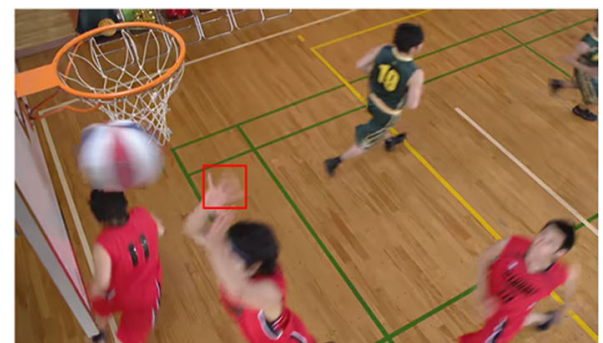

(a) encoded by the original HM 14.0

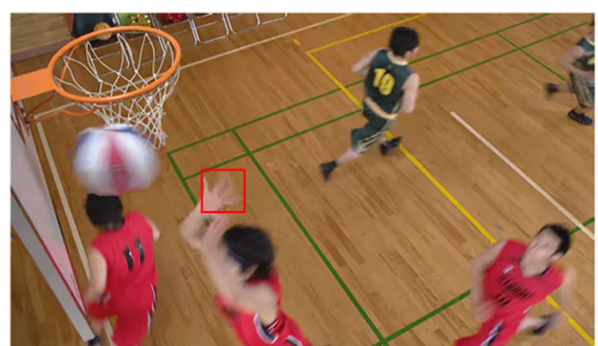

(b) encoded by the proposed algorithm

Fig. 9 Comparison of the subjective quality of the 32nd frame in test sequence "BasketballDrill". a Encoded by the original HM 14.0. b Encoded by the proposed algorithm

versus the original HM 14.0. From Table 8, we can note that sub-algorithm ADS can obtain coding complexity reduction with almost the same encoding performance for all ten test sequences. More specifically, it reduces 10.42 and $11.56 \%$ coding time averagely for them under RA and LDP cases, respectively. The loss of PSNR for ADS is from $-0.08 \mathrm{~dB}$ ("BasketballDrive" under LDP configuration) to $-0.02 \mathrm{~dB}$, and bitrate increase is from $0.42 \%$ ("BQSquare" under RA configuration) to $0.91 \%$ ("BasketballDrive" under LDP configuration). Since ADS simplifies AMP decision for PU mode decision, for the test sequences with high movement region, coding time saving is from the maximum of $14.33 \%$ ("BasketballDrive" at LDP condition) to the minimum of $12.53 \%$ ("RaceHorses" at LDP condition), which is evident. Although some of these ten test sequences have much slow motion areas (including "Traffic," "Cactus," "BQMall," "BQSquare,"“FourPeople," and "Johnny"), the coding time reduction still achieves approximately $8.67 \%$ under RA case and $10.43 \%$ under LDP case. Because of high definition and high motion activity, $\mathrm{RD}$ performance for test sequence "BasketballDrive" is less than satisfactory, but coding time saving reaches the expected goal with the maximum of $14.33 \%$ at LDP condition. These results imply that the proposed ADS algorithm can accelerate PU partition decision in HEVC encoder through skipping some AMP decision.

\subsection{Evaluation of individual algorithm SRA compared with HM 14.0}

Table 9 demonstrates the experimental results of our SRA algorithm compared with the original HM 14.0. It can be seen from Table 9 that over 16\% of time saving is obtained by SRA algorithm at both RA and LDP conditions. Meanwhile, decrease of PSNR and increase of bitrates are within acceptable range $(-0.02 \mathrm{~dB}$ of PSNR decrease and $0.37 \%$ of bitrates increase at RA condition as well as $0.01 \mathrm{~dB}$ of PSNR decrease and $0.28 \%$ of bitrates increase at LDP condition on average). In particular, for test sequences "BQSquare" with RA configuration and "Johnny" with LDP configuration, coding performance are totally identical to that of the original HEVC encoder in respect of PSNR. Additionally, bitrates rise from just $0.20 \%$ ("BQSquare" under LDP case) to $0.40 \%$ ("BQMall" under RA case) using SRA method. Because SRA adaptively diminishes the motion search range of ME and discards lots of unnecessary search region, bitrate increase is

Table 9 Comparison between SRA and the original HM 14.0

\begin{tabular}{|c|c|c|c|c|c|c|}
\hline \multirow[t]{2}{*}{ Test Sequence } & \multicolumn{3}{|l|}{ RA } & \multicolumn{3}{|l|}{ LDP } \\
\hline & BD-PSNR (dB) & BD-BR (\%) & $\overline{\Delta T(\%)}$ & BD-PSNR (dB) & BD-BR (\%) & $\Delta T(\%)$ \\
\hline Traffic & -0.02 & 0.39 & -15.52 & N/A & N/A & $\mathrm{N} / \mathrm{A}$ \\
\hline PeopleOnStreet & -0.01 & 0.37 & -17.28 & N/A & N/A & N/A \\
\hline BasketballDrive & -0.02 & 0.29 & -19.36 & -0.02 & 0.38 & -18.70 \\
\hline Cactus & -0.02 & 0.32 & -16.40 & -0.01 & 0.27 & -15.56 \\
\hline BQMall & -0.03 & 0.40 & -15.07 & -0.01 & 0.23 & -14.85 \\
\hline BasketballDrill & -0.01 & 0.28 & -18.44 & -0.02 & 0.36 & -19.44 \\
\hline BQSquare & -0.00 & 0.37 & -13.87 & -0.01 & 0.20 & -13.28 \\
\hline RaceHorses & -0.02 & 0.27 & -19.13 & -0.02 & 0.31 & -17.73 \\
\hline FourPeople & N/A & N/A & N/A & -0.01 & 0.26 & -16.51 \\
\hline Johnny & N/A & N/A & N/A & -0.00 & 0.25 & -16.18 \\
\hline Average & -0.02 & 0.37 & -16.88 & -0.01 & 0.28 & -16.53 \\
\hline
\end{tabular}


Table 10 Comparison between ADS + SRA and the original HM 14.0

\begin{tabular}{|c|c|c|c|c|c|c|}
\hline \multirow[t]{2}{*}{ Test Sequence } & \multicolumn{3}{|l|}{ RA } & \multicolumn{3}{|l|}{ LDP } \\
\hline & BD-PSNR (dB) & BD-BR (\%) & $\Delta T(\%)$ & BD-PSNR (dB) & BD-BR (\%) & $\Delta T(\%)$ \\
\hline Traffic & -0.01 & 0.28 & -29.13 & N/A & N/A & $\mathrm{N} / \mathrm{A}$ \\
\hline PeopleOnStreet & 0.00 & 0.25 & -27.24 & N/A & N/A & N/A \\
\hline BasketballDrive & -0.01 & 0.23 & -30.27 & -0.02 & 0.20 & -32.59 \\
\hline Cactus & 0.00 & 0.19 & -32.46 & 0.01 & 0.17 & -33.41 \\
\hline BQMall & 0.00 & 0.16 & -33.89 & 0.00 & 0.14 & -34.82 \\
\hline BasketballDrill & -0.01 & 0.20 & -31.69 & -0.01 & 0.15 & -32.35 \\
\hline BQSquare & 0.01 & 0.13 & -35.58 & 0.01 & 0.11 & -35.79 \\
\hline RaceHorses & -0.01 & 0.18 & -30.72 & 0.01 & 0.16 & -34.36 \\
\hline FourPeople & N/A & N/A & N/A & 0.00 & 0.10 & -35.03 \\
\hline Johnny & N/A & N/A & N/A & 0.01 & 0.09 & -37.21 \\
\hline Average & 0.00 & 0.20 & -31.37 & 0.00 & 0.14 & -34.45 \\
\hline
\end{tabular}

inconspicuous with regard to the test sequences with low motion activity areas, such as "BQSquare," "FourPeople," and "Johnny." It indicates that SRA is suitable for the test sequence whose objects move slowly, since SRA method can eliminate much computational complexity on candidate point searching through adjusting search range. Furthermore, coding time reduction is particularly significant on test sequences with complex movement, such as "BasketballDrive," "BasketballDrill," and "RaceHorses." The analysis about the results in Table 9 states that the proposed fast SRA algorithm maintains the nearly same coding efficiency as the original HEVC encoder with considerable reduction for the computational load.

\subsection{Proposed overall algorithm assessment}

Table 10 illustrates the experimental result comparison between the proposed overall algorithms (ADS plus SRA) and the original HEVC encoder. The results demonstrate that the proposed algorithm can save the encoding time dramatically with $31.37 \%$ under RA configuration and $34.45 \%$ under LDP configuration on average, where the maximum reduction of $37.21 \%$ ("Johnny" at LDP condition) and the minimum reduction of 27.24\% ("PeopleOnStreet" at RA condition), respectively.
When ADS performs, inter partition mode can be decided in advance. This process has accelerated intermode decision. Based on this improvement, ME can be skipped conditionally or performed under proper search range with SRA. Only performing ADS can skip some interpartition modes without any RD cost calculation process, but ME with full search range, if any, consumes much time. Similarly, although SRA can adjust search range for ME, computational complexity is still high during interpartition mode decision without ADS. It is inevitable that collaboration of ADS and SRA outperforms the individual algorithms. Therefore, the coding time reduction by joint algorithm is more than the time saved by ADS and SRA, respectively (11 and 16\%).

Test sequence "Johnny" has both large static background regions and large homogeneous areas, so most of CUs can be immediately predicted as SKIP mode without AMP decision using ADS. Furthermore, search range can be diminished as minimum range $[-8,8]$ using SRA for some blocks, which accomplishes coding time decrease efficiently. Since the proposed overall algorithms skip complicated AMP decision and adjust motion search range of ME process rather than exhaustive candidates search, the computational burden for the

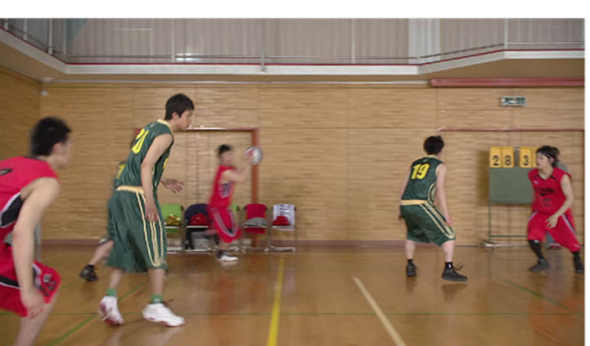

(a) encoded by the original HM 14.0

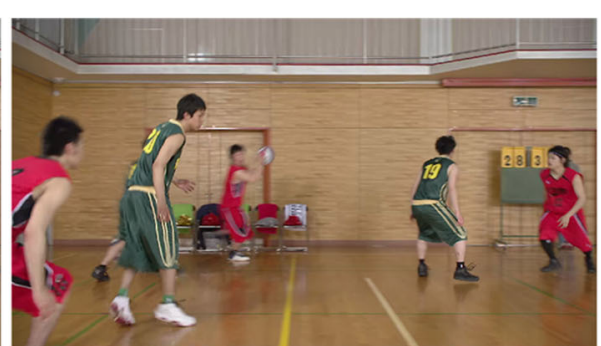

(b) encoded by the proposed algorithm

Fig. 10 Comparison of the subjective quality of the 42nd frame in test sequence "BasketballDrive" a Encoded by the original HM 14.0. b Encoded by the proposed algorithm 


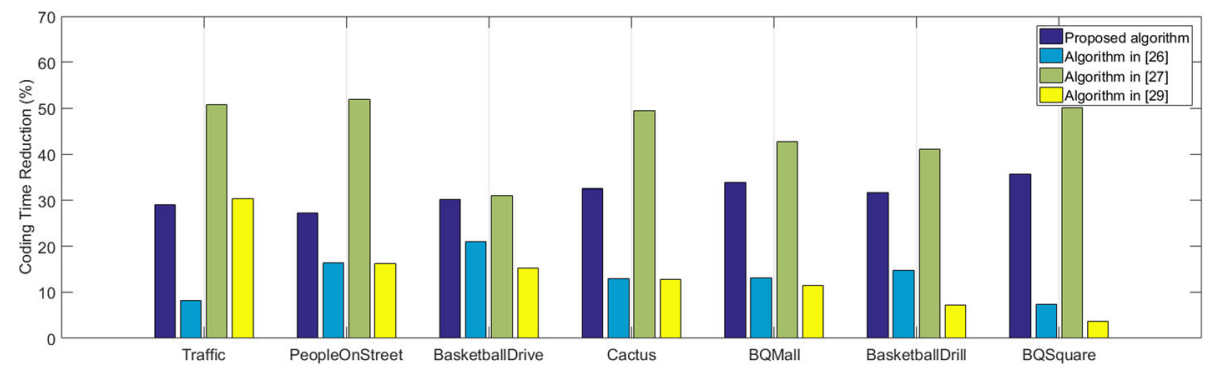

Fig. 11 Coding time reduction comparison of the proposed algorithm and the algorithms in [26, 27, 29] in RA case

sequences with low motion activity reduces significantly. For the sequences with slow movement, including "Traffic," "Cactus," "BQMall," "BQSquare," "FourPeople," and "Johnny," the proposed overall algorithms averagely save coding time $32.77 \%$ (in RA case) and 35.25\% (in LDP case), and bitrate increase for these sequences are only $0.19 \%$ (in RA case) and $0.12 \%$ (in LDP case) on average, which is negligible. Moreover, the average PSNR loss for all the test sequences is $0.00 \mathrm{~dB}$ under two configurations. These results indicate that the proposed efficient AMP decision and search range adjustment algorithm can accelerate intercoding greatly with the same coding performance as HEVC encoder.

\subsection{Intuitive evaluation of our proposed algorithm}

In order to illustrate the experimental results intuitively, Figs. 6 and 7 provide more information about the proposed overall algorithm compared with the original HEVC encoder in detail. It can be noticed from Figs. 6 and 7 that the proposed overall algorithm can obtain nearly the same coding performance as the original HEVC encoder in terms of PSNR and bitrates.

\subsection{Subjective performance comparison}

Figures 8, 9, and 10 show the 22nd frame of "RaceHorses" $(416 \times 240)$, the 32nd frame of "BasketballDrill" $(832 \times 480)$, and the 42nd frame of "BasketballDrive" $(1920 \times 1080)$ under RA case with QP of 32 , aiming at comparing subjective performance between our proposed approach and the original HEVC encoder.

It is evident that the region marked by the red rectangle in Fig. $8 \mathrm{~b}$ is more smoothly than that in Fig. 8a. Additionally, the area labeled by the red rectangle in Fig. 9a is more blurred than that in Fig. 9b. Except for Figs. 8 and 9, we can also find from Fig. 10 that there exists no apparent flaw in Fig. $10 \mathrm{~b}$ for the proposed algorithm from the perspective of subjective quality. The subjective performance comparison demonstrates that the proposed algorithm and the original HEVC encoder have extremely similar subjective qualities, but the coding efficiency of the proposed algorithm is higher than that of the original HEVC encoder.

\subsection{Comparison with the state-of-the-art algorithms [26,} 27, 29]

It is illustrated as Figs. 11, 12, and 13 that the coding efficiency comparison between the proposed algorithm and the state-of-the-art algorithms in Ref. [26, 27, 29] under RA configuration and Figs. 11, 12, and 13 reflect the comparisons about coding time reduction, Bjontegaard Delta PSNR, and Bjontegaard Delta Bitrate, respectively. From Fig. 11, it is obvious that other than the algorithm in [27], the coding time is generally saved more by the proposed algorithm than that by algorithms in [26, 29], but the bitrate increase by our method is significantly less than that by the method in [27] (as shown in Fig. 13). Meanwhile, according to the

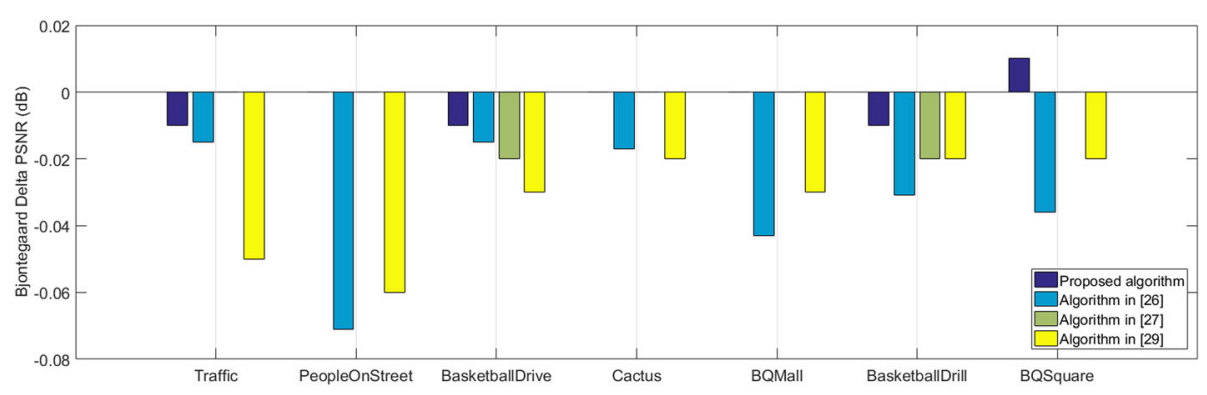

Fig. 12 PSNR change comparison of the proposed algorithm and the algorithms in $[26,27,29]$ in RA case (the blank is zero that means no change) 


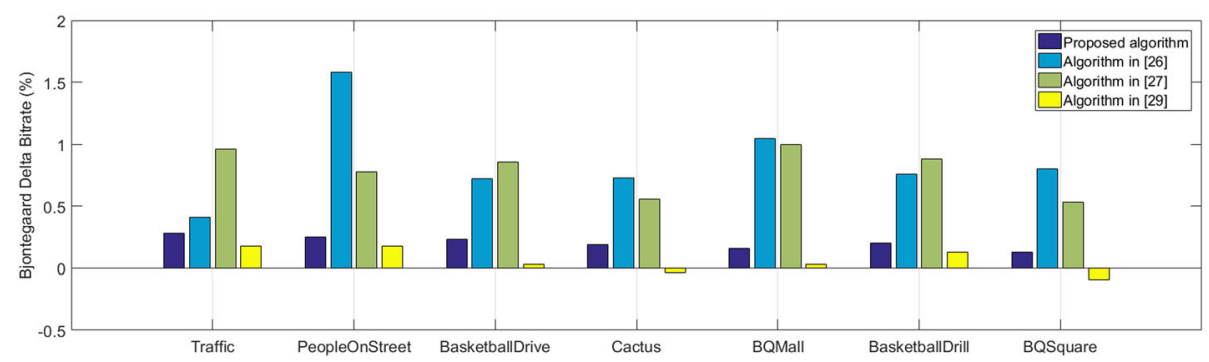

Fig. 13 Bitrate increase comparison of the proposed algorithm and the algorithms in $[26,27,29]$ in RA case

data comparisons from Fig. 12, we can see that in respects of PSNR loss achievement of our proposed algorithm is nearly same as that of algorithm in [27]. Note that the proposed algorithm distinctly outperforms the other algorithms in [26, 29]. For bitrate drop, our method is superior to both algorithms in $[26,27]$ except for algorithm in [29], while our approach achieves a better coding time reduction and PSNR gain than the approach in [29]. To sum up, the proposed algorithm can keep a consistent coding efficiency compared with the state-of-the-art fast HEVC algorithms in $[26,27,29]$.

\section{Conclusion}

In this paper, our proposed algorithm performs the outstanding coding efficiency for interprediction and is demonstrated by aforementioned experimental results. Because the proposed method achieves redundant PU mode decision skipping and adaptive search range of ME adjustment, it can reduce abundant coding time extremely and maintain almost the same coding performance as the original HEVC encoder. Moreover, comparison experiments with the state-of-the-art algorithms in Ref. [26, 27, 29] show that our algorithm is more close to the original HEVC encoder than other novel methods in terms of RD performance. In conclusion, the proposed algorithm can improve coding efficiency significantly and implement HEVC encoder for real-time application.

\section{Acknowledgements}

The authors would like to thank the editors and anonymous reviewers for their valuable comments.

\section{Funding}

This work was supported in part by the National Natural Science Foundation of China, under Grants U1301257, 61571285, and 61771432.

\section{Availability of data and materials}

Data will not be shared; reason for not sharing the data and materials is that the work submitted for review is not completed. The research is still ongoing, and those data and materials are still required by the author and co-authors for further investigations.

\section{Authors' contributions}

PA designed and conceived the research. XP performed the simulated experiments and QZ analyzed the exprimental results. XP wrote the manuscript. PA and QZ edited the manuscript. All authors read and approved the final manuscript.

\section{Authors' information}

Xinpeng Huang received the M.D. degree in information engineering from Zhengzhou University of Light Industry, Zhengzhou, China, in 2013. He is currently pursuing the Ph.D. degree in communication and information systems from Shanghai University, Shanghai, China. His current research interests include video coding, light field image compression, and extensions of the High Efficiency Video Coding.

Ping An received her B.S. and M.S. degrees from Hefei University of Technology, Hefei, China, in 1990 and 1993, respectively, and the Ph.D. degree in communication and information systems from Shanghai University, Shanghai, China, in 2002. She is currently a professor in School of Communication and Information Engineering, Shanghai University. Her research interests include stereoscopic and three-dimensional vision analysis and image and video processing, coding, and application.

Qiuwen Zhang received his Ph.D. degree in communication and information systems from Shanghai University, Shanghai, China, in 2012. Since 2012, he has been with the faculty of the College of Computer and Communication Engineering, Zhengzhou University of Light Industry, where he is currently an Associate Professor. He has published over 30 technical papers in the field of video coding and image processing. His major research interests include 3D signal processing, 3D High Efficiency Video Coding (3D-HEVC), video codec optimization, and multimedia communication.

\section{Competing interests}

The authors declare that they have no competing interests.

\section{Publisher's Note}

Springer Nature remains neutral with regard to jurisdictional claims in published maps and institutional affiliations.

\section{Author details}

${ }^{1}$ Shanghai Institute for Advanced Communication and Data Science, Shanghai University, Shanghai 200444, China. ${ }^{2}$ Key Laboratory of Ministry of Education for Advanced Display and System Application, Shanghai University, Shanghai 200072, China. ${ }^{3}$ College of Computer and

Communication Engineering, Zhengzhou University of Light Industry,

Zhengzhou 450002, China.

Received: 29 March 2017 Accepted: 7 November 2017

Published online: 21 November 2017

References

1. B Bross, W-JH, GJ Sullivan, J-R Ohm, T Wiegand. High Efficiency Video Coding (HEVC) text specification draft 8, in document JCTVC-J1003, Stockholm, Switzerland, 2012. http://phenix.intevry.fr/jct/doc_end_user/ documents/10_Stockholm/wg11/JCTVC-J1003-v8.zip.

2. GJ Sullivan, J-R Ohm, W-J Han, T Wiegand, Overview of the high efficiency video coding (HEVC) standard. IEEE Transactions on circuits and systems for video technology 22(12), 1649-1668 (2012)

3. G Corrêa, P Assunção, L Agostini, LADS Cruz, Performance and Computational Complexity Assessment of HEVC (Springer International 
Publishing, Cham, Switzerland, 2016). https://ink.springer.com/content/pdf/ 10.1007\%2F978-3-319-25778-5.pdf.

4. Rao, K.R.: High efficiency video coding. 2016 Signal Processing: Algorithms, Architectures, Arrangements and Applications (SPA), 11-11 (2016). doi:10. 1109/spa.2016.7763576

5. Mora EG, Cagnazzo M, Dufaux F. AVC to HEVC transcoder based on quadtree limitation. Multimedia Tools \& Applications 76(6), 1-25 (2017)

6. G Tech, Y Chen, K Müller, J-R Ohm, A Vetro, Y-K Wang, Overview of the multiview and 3D extensions of High Efficiency Video Coding. IEEE Transactions on Circuits and Systems for Video Technology 26(1), 35-49 (2016)

7. DA Milovanovic, D Kukolj, ZS Bojkovic, Recent Advances on 3D Video Coding Technology: HEVC Standardization Framework (Springer New York, New York, USA, 2017). https://link.springer.com/content/pdf/10.1007\%2F978-1-49394026-4.pdf.

8. F Bossen, B Bross, K Suhring, D Flynn, HEVC complexity and implementation analysis. IEEE Transactions on Circuits \& Systems for Video Technology 22(12), 1685-1696 (2012)

9. Yu, H., Wang, W., Xu, M.: Context Reduction of Palette Run Type in High Efficiency Video Coding (HEVC) Screen Content Coding (SCC). (2017).

10. P Wen-Hsiao, FG Walls, RA Cohen, X Jizheng, J Ostermann, A Maclnnis, L Tao, Overview of screen content video coding: technologies, standards, and beyond. IEEE J. Emerg. Sel. Top. Circuits Syst. (USA) 6(4), 393-408 (2016). doi: 10.1109/jetcas.2016.2608971

11. W Liu, J Li, YB Cho, A novel architecture for parallel multi-view HEVC decoder on mobile device. EURASIP Journal on Image and Video Processing 2017(1), 24 (2017). doi:10.1186/s13640-017-0174-5

12. Y Dai, D Liu, F Wu, A Convolutional Neural Network Approach for PostProcessing in HEVC Intra Coding (Springer International Publishing, Cham, Switzerland, 2017). https://link.springer.com/content/pdf/10.1007\%2F978-3319-51811-4.pdf.

13. A BenHajyoussef, T Ezzedine, A Bouallègue, Gradient-based pre-processing for intra prediction in High Efficiency Video Coding. EURASIP Journal on Image and Video Processing 2017(1), 9 (2017). doi:10.1186/s13640-016-0159-9

14. Roh HJ, Han SW, Ryu ES. Prediction complexity-based HEVC parallel processing for asymmetric multicores, 1-14, (2017). doi: 10.1007/s11042-0174413-7.

15. Zupancic, I, Blasi SG, Peixoto E, Izquierdo E. HEVC encoder optimisations using adaptive coding unit visiting order. In: IEEE International Conference on Image Processing 2016, pp. 794-798. http://ieeexplore.ieee.org/ document/7532466/.

16. Qing A, Zhou W, Wei H, Zhou X, Zhang G, Yang J. A fast CU partitioning algorithm in HEVC inter prediction for HD/UHD video. In: 2016 Asia-Pacific Signal and Information Processing Association Annual Summit and Conference (APSIPA), pp. 1-5. http://ieeexplore.ieee.org/document/7820718/.

17. Z Liu, X Yu, Y Gao, S Chen, X Ji, D Wang, CU partition mode decision for HEVC hardwired intra encoder using convolution neural network. IEEE Trans. Image Process. 25(11), 5088-5103 (2016). doi:10.1109/TIP.2016.2601264

18. G Correa, PA Assuncao, LV Agostini, LA da Silva Cruz, Fast HEVC encoding decisions using data mining. IEEE Transactions on Circuits and Systems for Video Technology 25(4), 660-673 (2015)

19. Zeng $\mathrm{H}$, Xiang W, Cai C, Chen J. Hierarchical inter mode decision for HEVC In: International Symposium on Intelligent Signal Processing and Communication Systems 2015, pp. 658-662. http://ieeexplore.ieee.org/ document/7432853/.

20. W Zhao, T Onoye, T Song, Hierarchical structure-based fast mode decision for H.265/HEVC. IEEE Transactions on Circuits \& Systems for Video Technology 25(10), 1651-1664 (2015)

21. Zupancic, I., Blasi, S.G., Peixoto, E., Izquierdo, E.: Inter-prediction optimizations for video coding using adaptive coding unit visiting order. IEEE Transactions on Multimedia PP(99), 1-1 (2016). doi:10.1109/TMM.2016.2579505

22. HS Kim, RH Park, Fast CU partitioning algorithm for HEVC using an onlinelearning-based Bayesian decision rule. IEEE Transactions on Circuits and Systems for Video Technology 26(1), 130-138 (2016). doi:10.1109/TCSVT. 2015.2444672

23. X Huang, Q Zhang, X Zhao, W Zhang, Y Zhang, Y Gan, Fast inter-prediction mode decision algorithm for HEVC. Signal Image Video Process. 11(1), 3340 (2017). https://doi.org/10.1007/s11760-016-0887-4

24. Zhang, N., Fan, X., Zhao, D., Gao, W.: Merge mode for deformable block motion information derivation. IEEE Transactions on Circuits and Systems for Video Technology PP(99), 1-1 (2016). doi:10.1109/TCSVT.2016.2589818
25. Nguyen $T$, Nguyen P, Nguyen P, Dinh C. A novel search pattern for Motion Estimation in High Efficiency Video Coding. In: 2016 International Conference on Computer Communication and Informatics (ICCCI), pp. 1-6. http://ieeexplore.ieee.org/document/7479971/.

26. Z Pan, J Lei, Y Zhang, X Sun, Fast motion estimation based on content property for low-complexity H.265/HEVC encoder. IEEE Trans. Broadcast. 62, 1-10 (2016)

27. A Medhat, A Shalaby, MS Sayed, M Elsabrouty, Adaptive low-complexity motion estimation algorithm for high efficiency video coding encoder. IET Image Process. 10(6), 438-447 (2016)

28. Wu J, Guo B, Hou J, Yan Y, Jiang J. Fast CU encoding schemes based on merge mode and motion estimation for HEVC inter prediction. Ksii Transactions on Internet \& Information Systems, 10(3),1195-1211 (2016)

29. H Kibeya, F Belghith, MAB Ayed, N Masmoudi, Fast coding unit selection and motion estimation algorithm based on early detection of zero block quantified transform coefficients for high-efficiency video coding standard. IET Image Process. 10(5), 371-380 (2016)

30. Bossen F. Common test conditions and software reference configurations, in document JCTVC-F900 Geneva, Switzerland, March, 2011. http://phenix. intevry.fr/jct/doc_end_user/documents/6_Torino/wg11/JCTVC-F900-v1.zip.

31. G Bjøntegaard. Calculation of average PSNR differences between RD-curves, ITU-T Q.6/SG16 VCEG 13th Meeting, Document VCEG-M33, Austin, USA, 2001

32. J Xiong, H Li, F Meng, S Zhu, Q Wu, B Zeng, MRF-based fast HEVC inter CU decision with the variance of absolute differences. IEEE Transactions on Multimedia 16(8), 2141-2153 (2014). doi:10.1109/TMM.2014.2356795

33. L Shen, Z Liu, Z Zhang, X Shi, Fast inter mode decision using spatial property of motion field. IEEE Transactions on Multimedia 10(6), 1208-1214 (2008). doi:10.1109/TMM.2008.2001358

34. L Shen, Z Zhang, Z Liu, Adaptive inter-mode decision for HEVC jointly utilizing inter-level and spatiotemporal correlations. IEEE Transactions on Circuits and Systems for Video Technology 24(10), 1709-1722 (2014). doi:10 1109/TCSVT.2014.2313892

35. Q Zhang, M Chen, X Huang, N Li, Y Gan, Low-complexity depth map compression in HEVC-based 3D video coding. EURASIP Journal on Image and Video Processing 2015(1), 2 (2015). doi:10.1186/s13640-015-0058-5

\section{Submit your manuscript to a SpringerOpen ${ }^{\circ}$ journal and benefit from:}

- Convenient online submission

- Rigorous peer review

- Open access: articles freely available online

- High visibility within the field

- Retaining the copyright to your article

Submit your next manuscript at $\boldsymbol{\nabla}$ springeropen.com 